\title{
Reluctant Vesicles Contribute to the Total Readily Releasable Pool in Glutamatergic Hippocampal Neurons
}

\author{
Krista L. Moulder ${ }^{1}$ and Steven Mennerick ${ }^{1,2}$ \\ Departments of ${ }^{1}$ Psychiatry and ${ }^{2}$ Anatomy and Neurobiology, Washington University School of Medicine, St. Louis, Missouri 63110
}

\begin{abstract}
The size of the readily releasable pool (RRP) of vesicles is critically important for determining the size of postsynaptic currents generated in response to action potentials. However, discrepancies in RRP estimates exist among methods designed to measure RRP size. In glutamatergic hippocampal neurons, we found that hypertonic sucrose application yielded RRP size estimates approximately fivefold larger than values obtained with high-frequency action potential trains commonly assumed to deplete the RRP. This discrepancy was specific for glutamatergic neurons, because no difference was found between sucrose and train estimates of RRP size in GABAergic neurons. A small component of the difference in excitatory neurons was accounted for by postsynaptic receptor saturation. Train estimates of vesicle pool size obtained using more stimuli revealed that action potential-elicited EPSCs did not truly reach a steady state during shorter trains, and RRP estimates were closer to sucrose estimates made in the same neurons. This suggested that reluctant vesicles may contribute to the total available pool. Two additional lines of evidence supported this hypothesis. First, RRP estimates from strongly depolarizing hyperkalemic solutions closely matched those obtained with sucrose. Second, when $\mathrm{Ca}^{2+}$ influx was enhanced during trains, train estimates of pool size matched those obtained with sucrose. These data suggest that glutamatergic hippocampal neurons maintain a heterogeneous population of vesicles that can be differentially released with varying $\mathrm{Ca}^{2+}$ influx, thereby increasing the range of potential synaptic responses.
\end{abstract}

Key words: glutamate; voltage-clamp; readily releasable pool; probability of release; calcium; excitatory synaptic transmission

\section{Introduction}

The size of postsynaptic currents (PSCs) generated in response to synaptic vesicle release is dependent on the quantal size $(q)$, the number of release sites $(N)$, and the probability of vesicle release $\left(p_{\mathrm{r}}\right)($ Katz, 1969; Johnson and Wernig, 1971; Zucker, 1973; Quastel, 1997). Although many meanings have been ascribed to the value $N$, one commonly accepted definition is that $N$ corresponds to the total readily releasable pool (RRP) of vesicles (for review, see Schneggenburger et al., 2002). However, the nature of the RRP remains controversial. Experiments to define the RRP typically use a stimulus to deplete the RRP and measure the resulting postsynaptic response. Frequently used depletion techniques include sustained action potential activity (Rosenmund and Stevens, 1996; Wang and Kaczmarek, 1998; Schneggenburger et al., 1999), strong direct depolarization (Sun and Wu, 2001; Mozhayeva et al., 2002), and hypertonic sucrose solution application (Rosenmund and Stevens, 1996). At issue is whether each of these techniques measures the depletion of the same pool of available vesicles. For example, recent work at the calyx of Held has revealed a large discrepancy between RRP size estimates de-

Received Dec. 22, 2004; revised Feb. 21, 2005; accepted March 8, 2005.

This work was supported by National Institutes of Health Grants AA12592 and NS40488 (S.M.) and F32DA15948 and K01DA18109 (K.M.). We thank Ann Benz for preparation of hippocampal cultures and members of the laboratory for advice and criticism. We also thank Charles Zorumski for advice and support.

Correspondence should be addressed to Dr. Steven Mennerick, Department of Psychiatry, Washington University School of Medicine, 660 South Euclid Avenue, Box 8134, St. Louis, M0 63110. E-mail: menneris@psychiatry.wustl.edu. DO1:10.1523/JNEUROSC1.5231-04.2005

Copyright $\odot 2005$ Society for Neuroscience $\quad 0270-6474 / 05 / 253842-09 \$ 15.00 / 0$ rived from action potential trains and estimates from strong depolarizing stimuli (Schneggenburger et al., 2002). In contrast, at small hippocampal synapses, action potential trains have been proposed to deplete the same pool of vesicles depleted by strong hypertonic challenge (Rosenmund and Stevens, 1996; Otsu et al., 2004). Whether these discrepancies result from the methods used to evaluate the RRP size or whether they result from differences inherent to different synaptic types is not known.

Contrary to previous studies at hippocampal synapses (Rosenmund and Stevens, 1996), our measurements suggest a large discrepancy between two standard methods of "depleting" glutamate synapses: action potential trains and hypertonic sucrose application. Charge integrals of sucrose-evoked EPSCs were approximately fivefold larger than cumulative charge estimates derived from $20 \mathrm{~Hz}$ action potential-evoked EPSC trains, a protocol commonly used to deplete the RRP (Murthy and Stevens, 1998, 1999; Schikorski and Stevens, 2001; Moulder et al., 2004). The difference observed with train estimates of RRP size only partially resulted from postsynaptic factors such as glutamate receptor saturation, suggesting a presynaptic explanation for the RRP discrepancy. RRP estimates using strong depolarization matched sucrose estimates of RRP, consistent with the idea that sucrose application accessed all readily releasable vesicles, regardless of their individual $p_{\mathrm{r}}$ value. Increased $\mathrm{Ca}^{2+}$ influx during trains also eliminated the discrepancy between sucrose and train estimates.

We therefore conclude that commonly used RRP depletion protocols using action potential trains do not elicit full depletion 
of the RRP in glutamatergic hippocampal neurons. In addition, discrepancies between sucrose-elicited vesicle release and release resulting from high-frequency stimulation were observed in glutamatergic, but not GABAergic, neurons, suggesting that reluctant vesicles (Sun and $\mathrm{Wu}, 2001$ ) contribute to a heterogeneous RRP population selectively in glutamatergic hippocampal neurons. This spatially or temporally heterogeneous pool may contribute to added plasticity in excitatory neurons when faced with varying stimulus patterns.

\section{Materials and Methods}

Materials. Unless otherwise indicated, reagents were purchased from Sigma (St. Louis, MO).

Cell culture. Hippocampal cultures were prepared as described previously (Mennerick et al., 1995). In brief, dissected postnatal day 0 (P0)-P3 rat hippocampi were incubated with papain and then mechanically dissociated and plated at 100 cells $/ \mathrm{mm}^{2}$ on microdots of collagen. Plating medium consisted of Eagle's minimal essential medium (Invitrogen, Carlsbad, CA) supplemented with heat-inactivated horse serum (5\%), fetal bovine serum (5\%), $17 \mathrm{~mm}$ glucose, $400 \mu \mathrm{M}$ glutamine, $50 \mathrm{U} / \mathrm{ml}$ penicillin, and $50 \mu \mathrm{g} / \mathrm{ml}$ streptomycin. Cytosine arabinoside $(6.7 \mu \mathrm{M})$ was added 3-4 d after plating to inhibit cell division. At $4-5 \mathrm{~d}$ after plating, a half-volume medium replacement was conducted using Neurobasal medium (Invitrogen) plus a B27 supplement. Electrophysiological recordings were conducted 11-14 d after plating.

Electrophysiology. Whole-cell recordings of excitatory or inhibitory currents were performed on solitary hippocampal neurons, thus excluding polysynaptic contributions to measured responses. Recordings were made using an Axopatch 1D amplifier (Axon Instruments, Union City, CA) and a Digidata 1200 acquisition board (Axon Instruments). Electrodes had resistances of 3-5 M $\Omega$, and access resistance was compensated $80-100 \%$. In all instances, cells were excluded from analysis if a leak current $>300 \mathrm{pA}$ was observed. No correlation between the amplitudes of leak currents $<300 \mathrm{pA}$ and the areas of RRP estimates from sucrose was found $\left(r^{2}=0.00 ; p=0.98\right)$ (Aravamudan et al., 1999). All recordings were performed at $22^{\circ} \mathrm{C}$.

For recording, the culture medium was exchanged for a saline solution containing the following (in mM): $138 \mathrm{NaCl}, 4 \mathrm{KCl}, 2 \mathrm{CaCl}_{2}, 1 \mathrm{MgCl}_{2}, 10$ glucose, 10 HEPES, and $0.025 \mathrm{D}-\mathrm{APV}, \mathrm{pH} 7.25$. In some experiments, the $\mathrm{CaCl}_{2}$ and $\mathrm{MgCl}_{2}$ concentrations were altered as indicated. The wholecell pipette solution contained the following (in $\mathrm{mM}$ ): $140 \mathrm{~K}$-gluconate, $0.5 \mathrm{CaCl}_{2}, 5$ EGTA, and 10 HEPES, pH 7.25. For GABAergic cells, $\mathrm{KCl}$ was used in the pipette solution in place of K-gluconate. For synaptic recordings, cells were stimulated with $1.5 \mathrm{~ms}$ pulses from -70 to $0 \mathrm{mV}$ to evoke transmitter release (Mennerick et al., 1995). We verified that allor-none EPSCs were generated by this presynaptic stimulation method. Pulses $(1.5 \mathrm{~ms})$ to potentials varying from -60 to $0 \mathrm{mV}$ yielded an all-or-none EPSC in all cells tested $(n=6 ; 0.5 \pm 1.3 \%$ difference between the smallest pulse yielding a measurable EPSC and the largest pulse delivered). Likewise, when conditioning stimulation was given to the standard $0 \mathrm{mV}$ and the amplitude of test stimulation ( $50 \mathrm{~ms}$ later) was varied, the test response was always all or none $(n=3 ; 1.4 \pm 1.2 \%$ difference in the amplitude of test EPSCs at the smallest and largest stimulus amplitudes). Therefore, variable clamp control over the presynaptic elements did not contribute to synaptic depression observed in the present studies.

Solutions were exchanged via a local multibarrel perfusion pipette with a common perfusion port placed within $0.5 \mathrm{~mm}$ of the cell under study. Liquid junction potential measurements exhibit exchange times between barrels of $\sim 50 \mathrm{~ms}$. For hypertonic solution ( $0.5 \mathrm{M}$ sucrose) or hyperkalemic solution (100 mM KCl; equimolar substitution for $\mathrm{NaCl}$ ) applications, drug was applied for $3 \mathrm{~s}$. Sucrose responses have been shown to be completely CNQX/2,3-dihydroxy-6-nitro-7-sulfonylbenzo[f] quinoxaline (NBQX) sensitive (Rosenmund and Stevens, 1996; Moulder et al., 2003). Sucrose responses were integrated to include responses beneath the transient peak of the response to $10 \%$ of the steadystate response. Digital subtraction of a trace in the presence of $1 \mu \mathrm{M}$ $\mathrm{NBQX}$ was used to isolate the AMPA receptor contribution to the hyperkalemic response. The peak NBQX-sensitive response was integrated

A

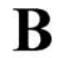

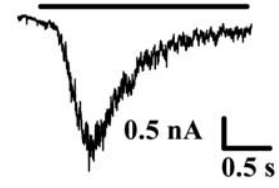

C
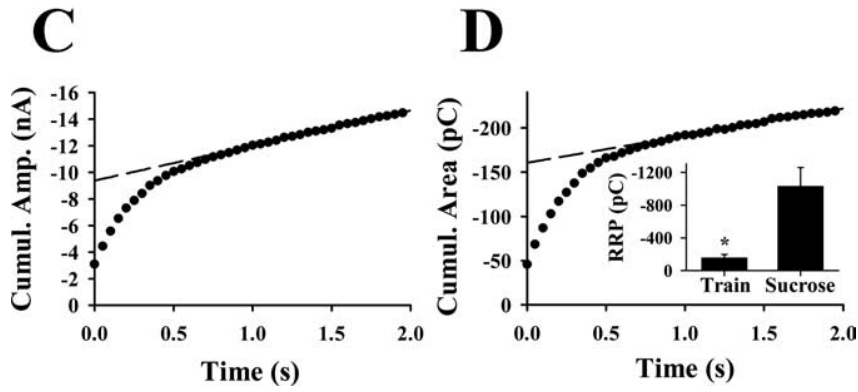

Figure 1. Estimates of RRP size using sucrose application and action potential trains do not match in glutamatergic neurons. $\boldsymbol{A}$, Representative response to exogenous application of $0.5 \mathrm{M}$ sucrose. In all figures, the horizontal bar denotes the time of exposure to the secretagogue. $\boldsymbol{B}$, Representative EPSC train (40 stimuli; $20 \mathrm{~Hz}$ ) recorded from the same neuron as in $\boldsymbol{A}$. Presynaptic stimulus transients have been blanked for clarity. C, Representative cumulative EPSC amplitude (Cumul. Amp.) values from a 40 stimuli, $20 \mathrm{~Hz}$ train. The dashed line represents a linear regression fit to data points after $1.5 \mathrm{~s}$ to estimate the cumulative amplitude at time $0 . \boldsymbol{D}$, Representative cumulative EPSC area values from the same 40 stimuli, $20 \mathrm{~Hz}$ train as in $\boldsymbol{C}$. The dashed line represents a linear regression fit to data points after $1.5 \mathrm{~s}$ to estimate the cumulative area at time 0 . Inset, Summary of the total RRP charge, as determined with trains (cumulative area at time 0$)$ and sucrose applications in the same neurons. ${ }^{*} p<0.002(n=8)$. Error bars represent SEM.

to include the time over which the peak decayed to $10 \%$ of the steadystate response; this integral represented our estimate of the RRP depleted by hyperkalemia. Recordings of EPSC trains made in the presence of 4-(8methyl-9H-1,3-dioxolo [4,5- $h]$ [2,3] benzodiazepin-5-yl)-benzenamine hydrochloride (GYKI 52466) were also NBQX subtracted to obtain accurate measurements of EPSC charge.

For IPSC trains, some IPSCs (approximately one-third) did not decay fully back to baseline within the $100 \mathrm{~ms}$ interpulse interval of the $10 \mathrm{~Hz}$ train (see Fig. 2). Therefore, to determine accurate measurements of IPSC cumulative area, a single IPSC was also recorded in those cells, and the fraction of the charge after $100 \mathrm{~ms}$ was then added to the charge measurement of every IPSC in the train.

Data acquisition and statistics. pClamp software, version 9 (Axon Instruments), was used for electrophysiology data acquisition and analysis for all experiments. Data plotting and curve fitting were done with SigmaPlot software (SPSS, Chicago, IL). Data are presented in the figures and text as mean \pm SEM. Paired and unpaired $t$ tests were used to evaluate statistical significance.

\section{Results}

\section{Estimating RRP size using hypertonic sucrose application or} action potential trains

To evaluate the degree of correspondence between two common methods of RRP depletion, hypertonic challenge and action potential trains, we used autaptic synapses so that we could challenge the same set of synaptic contacts with multiple depleting stimuli. In the same neurons, $0.5 \mathrm{M}$ sucrose was rapidly applied to solitary excitatory neurons (Rosenmund and Stevens, 1996), and an action potential train of 40 stimuli at $20 \mathrm{~Hz}$ was delivered (Murthy and Stevens, 1998; Schikorski and Stevens, 2001). Each of these protocols was designed to measure the total number of releasable vesicles. Examples of the currents elicited by sucrose application and action potential trains are shown in Figure 1, $A$ and $B$. Sucrose responses reached a peak and then decayed, de- 
spite the continued presence of sucrose (Fig. 1A). This decay to a steady-state response is thought to represent the depletion of the $\mathrm{RRP}$, and the steady state is thought to represent ongoing replenishment of the RRP. Therefore, the RRP is typically quantified by integrating the sucrose response under the transient peak (Reim et al., 2001).

EPSCs during the $20 \mathrm{~Hz}$ train, as has been observed previously, depressed and reached an apparent steady state over the course of 40 stimuli (Fig. $1 \mathrm{~B}$ ). The depression is typically interpreted to represent depletion of the RRP, with the steady state representing ongoing replenishment of the depleted RRP (Schneggenburger et al., 1999; Hagler and Goda, 2001; Otsu et al., 2004). To estimate the RRP from action potential trains, excluding the component representing replenishment, we plotted the cumulative EPSC amplitude during the action potential train and back extrapolated to time 0 to determine the cumulative amplitude of the EPSCs representing the RRP (Schneggenburger et al., 1999) (Fig. 1C).

When we compared RRP estimates, we found that the average sucrose charge was $-1034 \pm 224 \mathrm{pC}$, and the average cumulative amplitude was $-11.1 \pm 3.3 \mathrm{nA}(n=8)$. Given an mEPSC charge of $-81.5 \mathrm{fC}$ (Moulder et al., 2004) and an mEPSC amplitude of $-18.3 \mathrm{pA}$ (data not shown), these estimates correspond to 12,681 vesicles in the sucrose-depleted RRP and 606 vesicles in the traindepleted RRP. To account for asynchrony during action potential trains (Diamond and Jahr, 1995; Lu and Trussell, 2000; Hagler and Goda, 2001; Otsu et al., 2004), we made cumulative area plots from the $20 \mathrm{~Hz}$ trains (Fig. $1 \mathrm{D}$ ). The average cumulative area at time 0 was $-158 \pm 43 \mathrm{pC}(n=8)$. Although this represents $\sim 1945$ vesicles, a more than threefold increase in vesicles over the estimate from EPSC peak amplitudes, this still only corresponds to $15.34 \%$ of the sucrose estimate (Fig. $1 D$, inset).

We considered the possibility that sucrose estimates may be inflated by regenerative dendritic sodium currents. Although we confirmed the NBQX sensitivity of sucrose-evoked EPSCs, active currents could occur on poorly clamped AMPA receptormediated EPSCs and therefore might only be present when AMPA receptors are intact. We found no effect of tetrodotoxin on sucrose-evoked EPSCs $(9.2 \pm 5.2 \%$ change in sucrose-evoked RRP estimate; $n=5$ ), excluding this mechanism of an inflated sucrose estimate.

To test whether the RRP discrepancy is ubiquitous among different synaptic types, we challenged GABAergic solitary neurons with action potential trains and hypertonic solution. Twenty stimuli at $10 \mathrm{~Hz}$ were delivered to solitary inhibitory neurons to deplete the GABAergic RRP (Kirischuk and Grantyn, 2000) (Fig. $2 B, C)$, and the cumulative area at time 0 was compared with charge estimates from sucrose-evoked currents (Fig. $2 A$ ). Unlike in glutamatergic neurons, train estimates of RRP size were not statistically different from those obtained with sucrose $(p<0.23$; $n=10$ ) (Fig. 2D), suggesting that specific postsynaptic or presynaptic factors were contributing to RRP estimates in glutamatergic neurons. Although we used different stimulus frequencies for IPSC and EPSC stimulation to match existing literature (Rosenmund and Stevens, 1996; Kirischuk and Grantyn, 2000), we found no difference in action potential RRP estimates for EPSCs at 10 versus $20 \mathrm{~Hz}$ (data not shown).

We also considered that release-independent depression via action potential failure may contribute to the smaller action potential-derived estimates of RRP (Brody and Yue, 2000). Our own previous work has shown that EPSCs are more prone than GABAergic IPSCs to depression by sodium channel blockade/ inactivation. This mechanism could therefore potentially con-
A B

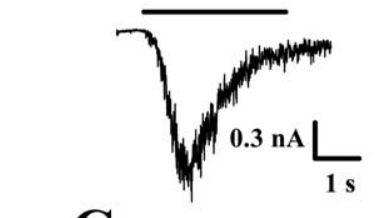

C

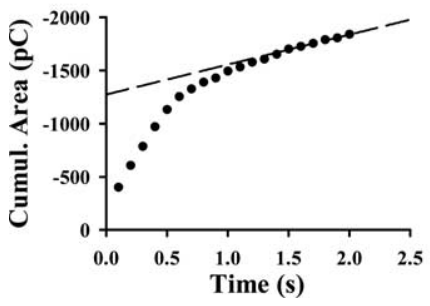

D
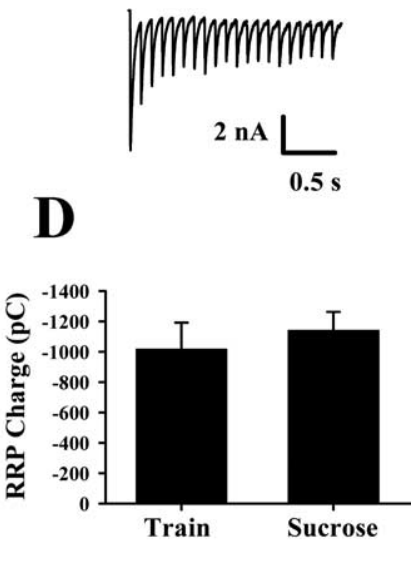

Figure 2. Train estimates of RRP size match sucrose estimates in GABAergic neurons. $\boldsymbol{A}$ Representative response to exogenous application of $0.5 \mathrm{~m}$ sucrose. $\boldsymbol{B}, A$ representative IPSC train $(20$ stimuli; $10 \mathrm{~Hz}$ ) recorded from the same neuron as in $\boldsymbol{A}$. Presynaptic stimulus transients have been blanked for clarity. C, Representative cumulative IPSC area (Cumul. Area) values from the same 20 stimuli, $10 \mathrm{~Hz}$ train. The dashed line represents a linear regression fit to data points after $1.5 \mathrm{~s}$ to estimate the cumulative area at time $0 . \boldsymbol{D}$, Summary of the total RRP charge, as determined with trains (cumulative area at time 0 ) and sucrose applications performed in the same neurons $(n=10)$. Error bars represent SEM.

tribute to the different behavior of glutamate and GABA synapses in the present experiments. To test the contribution of this mechanism, we compared action potential trains in $120 \mathrm{~mm}$ extracellular $\mathrm{Na}^{+}$with trains in the presence of elevated $\mathrm{Na}^{+}(160 \mathrm{mM})$ plus lowered extracellular divalents $\left(1.1 \mathrm{mM} \mathrm{Ca}^{2+}\right.$ and $0 \mathrm{~mm}$ $\left.\mathrm{Mg}^{2+}\right)$. Previously, we showed that either elevated $\mathrm{Na}^{+}$(He et al., 2001) or lowered divalents (Prakriya and Mennerick, 2000) alone significantly increase excitability in hippocampal neurons. In the present experiments, the combined effect of these manipulations failed to relieve the depression in the EPSC area observed during a 40 stimuli $20 \mathrm{~Hz}$ train. These results are consistent with our previous study suggesting that there is little effect of action potential failure on synaptic depression in these cells under normal conditions (He et al., 2001). Together with the results in Figures 1 and 2, these results suggest that factors contributing to glutamate RRP discrepancies do not arise from inherent discrepancies in the depletion methods used or in differences in action potential coupling to release. Rather, the RRP discrepancy apparently arises from mechanisms controlling presynaptic or postsynaptic glutamate function.

Assessment of postsynaptic factors in RRP size determination Because electrically evoked EPSCs are of a much greater amplitude than sucrose-evoked currents and are much more synchronous (Fig. $1 A, B$ ), we considered that train estimates of RRP size were confounded by postsynaptic factors including access resistance errors, receptor saturation, or receptor desensitization. Each of these factors could lead to an underestimate of the amount of glutamate released. To address the first concern, we examined sucrose responses and $20 \mathrm{~Hz}$ trains in glutamatergic neurons in the presence and absence of the noncompetitive AMPA receptor antagonist GYKI 52466. We found that the discrepancy in RRP estimates persisted in the presence of $25 \mu \mathrm{M}$ GYKI 52466, a concentration that inhibited EPSCs by $82.6 \%$ (Fig. $3 A, C)$, suggesting that train estimates of RRP size were not limited by access resistance errors. 
A

\section{B}
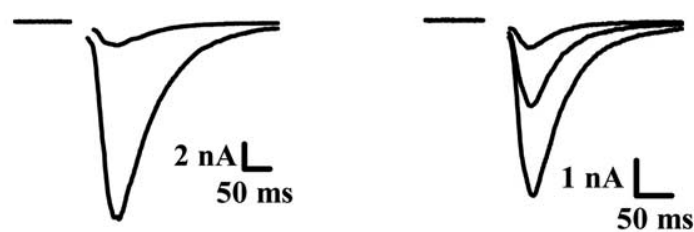

C

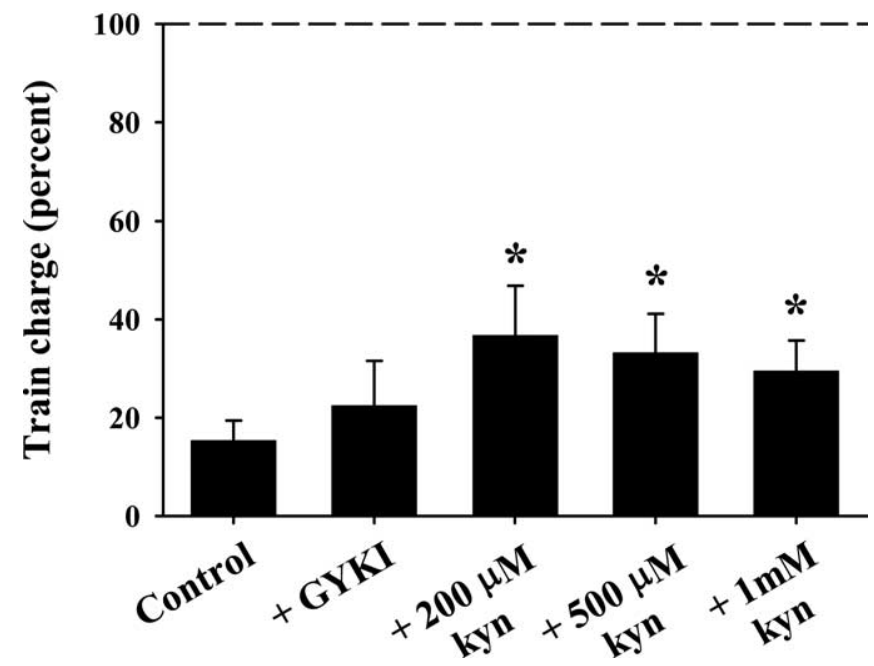

Figure 3. Postsynaptic factors contribute only slightly to the discrepancy in RRP estimates. $\boldsymbol{A}$, Representative EPSCs in the presence and absence of $25 \mu \mathrm{M}$ GYKI 52466. $\boldsymbol{B}$, Representative EPSCs in the presence of 0,200 , and $1000 \mu \mathrm{m}$ kynurenate. $\boldsymbol{A}, \boldsymbol{B}$, Presynaptic stimulus transients have been blanked for clarity. $C$, Summary of train estimates of RRP size, expressed as percentages of sucrose estimates of RRP size performed in the same neurons, in control conditions and in the presence of $25 \mu \mathrm{M}$ GYKI 52466 (GYKI) or kynurenate (kyn). ${ }^{*} p<0.03$ compared with train estimates made in control conditions; in all conditions, train estimates were significantly different from sucrose estimates made in the same neurons ( $n=5-13$ in each condition). Error bars represent SEM.

Recently, receptor saturation and desensitization have been shown to limit EPSCs at several glutamate synapses (Brenowitz and Trussell, 2001; Neher and Sakaba, 2001; Sun and Wu, 2001; Wadiche and Jahr, 2001; Foster et al., 2002; Xu-Friedman and Regehr, 2003). Because these studies have examined saturation and desensitization at specialized synaptic structures such as calyces and glomerular synapses, it is not clear to what degree receptor saturation limits EPSC amplitudes at small hippocampal synapses (Tong and Jahr, 1994). To investigate this possibility, we examined sucrose responses and $20 \mathrm{~Hz}$ trains in glutamatergic neurons in the presence and absence of the rapidly dissociating AMPA receptor antagonist kynurenate. We found that $200 \mu \mathrm{M}$ kynurenate, a concentration that inhibited the first EPSC in the train by $47.1 \%$ (Fig. $3 B$ ) and that should limit receptor saturation (Diamond and Jahr, 1997), reduced the discrepancy in RRP estimates from a 6.5-fold difference to a 2.7-fold difference (Fig. 3C). Increasing the kynurenate concentration to $1 \mathrm{~mm}$, which blocked $85.5 \%$ of initial EPSCs (Fig. 3B), failed to further decrease the discrepancy (Fig. $3 C$ ). Train estimates of RRP size made in the presence of kynurenate were statistically different from control estimates ( $p<0.03$ in all kynurenate conditions) but not statistically different from estimates made in the presence of GYKI 52466 ( $p>0.05$ ), suggesting only a minor contribution of saturation to the RRP discrepancy. We found that $100 \mu \mathrm{M}$ cyclothia- zide applied alone or in combination with kynurenate had no effect on action potential-elicited RRP size estimates (data not shown), suggesting that AMPA receptor desensitization did not contribute to the discrepancy in RRP size measurements in hippocampal neurons. Together, these results suggest that there are minor contributions of access resistance errors and postsynaptic saturation to the RRP discrepancy but that presynaptic factors explain the bulk of the discrepancy. To focus on the large presynaptic contribution to RRP estimate discrepancies, $200 \mu \mathrm{M}$ kynurenate was included in all subsequent measures of RRP size.

\section{EPSC trains deplete a smaller pool than sucrose-elicited vesicle release}

Because action potential trains yielded smaller estimates of RRP than sucrose-elicited vesicle release, we tested whether these procedures were indeed depleting the same pool of vesicles. If the same pool of vesicles was being tapped by each, a depleting train of action potentials should depress EPSCs and sucrose responses by the same amount (Rosenmund and Stevens, 1996). Therefore, in one set of experiments, we assayed the charge integral of an EPSC or the sucrose response, gave the cell a strong depleting action potential train of 100 pulses at $20 \mathrm{~Hz}$, and then reassayed the EPSC or the sucrose response at $0.5 \mathrm{~s}$ after the end of the depleting train. We found that EPSCs were significantly more depressed at $0.5 \mathrm{~s}$ after the stimulus train compared with sucrose responses (Fig. 4A). This result suggests that action potential trains may deplete only a subset of vesicles accessible to sucrose.

We considered the alternative possibility that the discrepancy in the depression of EPSCs versus sucrose responses (Fig. 4A) could also result if action potentials fully deplete the vesicles accessible to both stimuli but the pool accessible to sucrose recovers more quickly than the pool accessible to action potentials. To test this, we examined the recovery rates of EPSCs and sucrose responses after a $20 \mathrm{~Hz}$ train of 100 stimuli (Rosenmund and Stevens, 1996). We found that the charge of individual EPSCs and of sucrose responses recovered with approximately the same time course after the 100 stimuli (Fig. $4 B$ ). When fit with singleexponential recovery curves, sucrose responses actually recovered slightly more slowly than action potential responses (Fig. $4 B$ ), so recovery differences cannot account for the less-severe depression of sucrose responses. When a $20 \mathrm{~Hz}$ train of only 40 stimuli (identical to the protocol used in Figs. 1-3) was instead used as the depleting stimulus, sucrose responses were only inhibited $\sim 25 \%$ at $0.5 \mathrm{~s}$ (Fig. $4 A$, right bar), consistent with an approximate fourfold to sixfold difference in the RRP size estimates from the two methods.

These results suggest that action potentials deplete only a subset of the total number of vesicles accessible to sucrose. To determine whether the pool of vesicles accessible to sucrose completely overlaps that accessible to action potentials, we used sucrose as the depleting stimulus. We found that the charge of an individual EPSC and of a sucrose response recovered in parallel (Fig. 4C), even from the earliest time point. This is in contrast to what we had observed when an action potential train was used as the depleting stimulus, strengthening the idea that the sucrose application can evoke the release of a larger vesicle pool, one that completely encompasses the pool accessible to action potentials. Consistent with previous reports (Rosenmund and Stevens, 1996), we also observed that the recovery of both EPSCs and sucrose responses was faster after action potential trains (Fig. 4, compare $B$ and $C$ ), suggesting that the rise in the intracellular calcium concentration $\left(\left[\mathrm{Ca}^{2+}\right]_{\mathrm{i}}\right)$ that accompanies trains speeds 
A

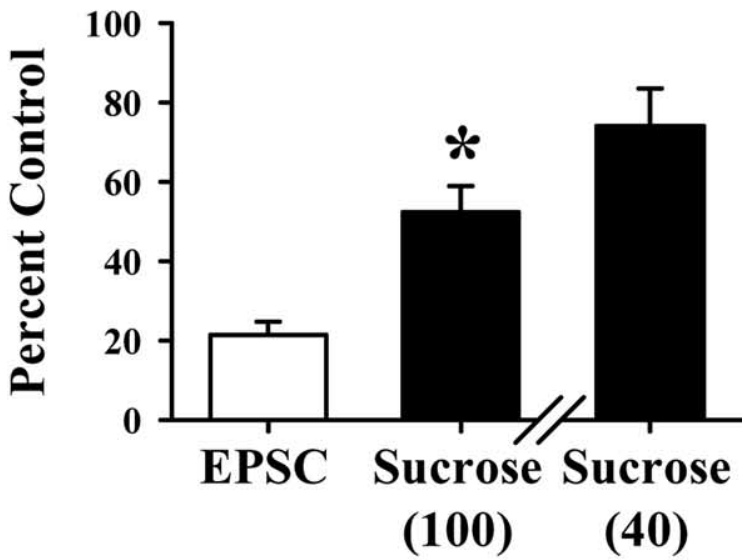

B
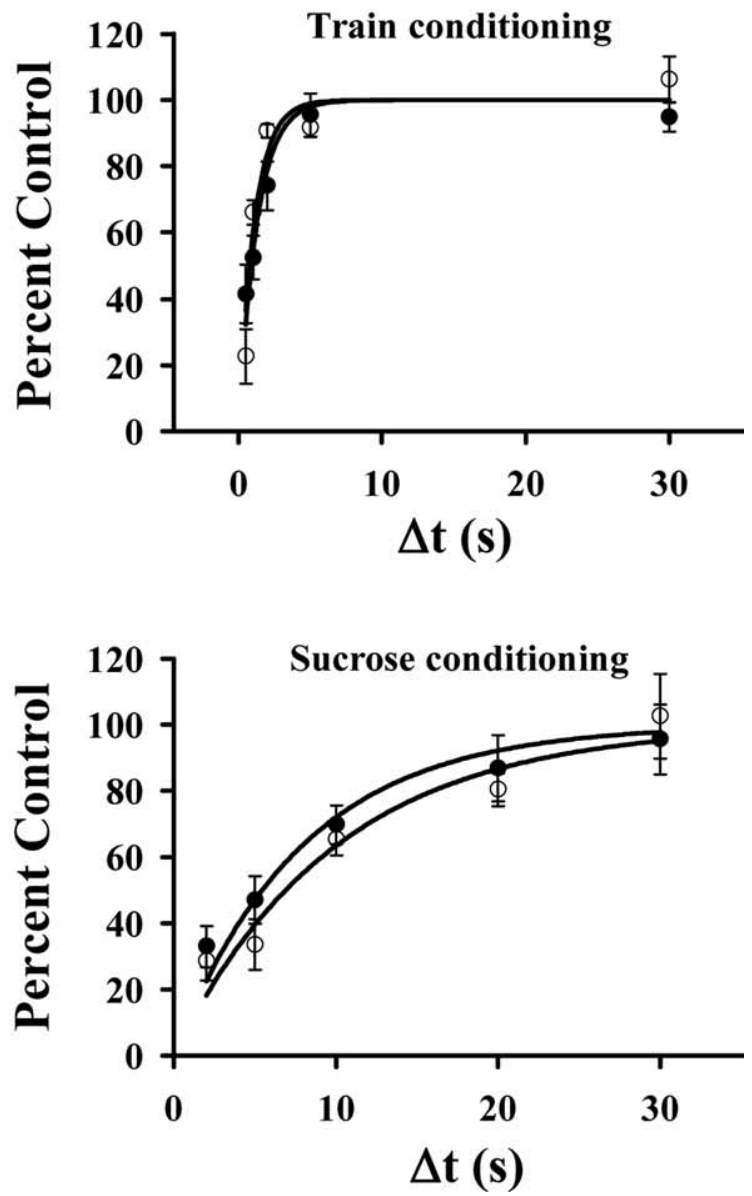

Figure 4. Cross-depletion experiments reveal that EPSC trains deplete only a subset of the sucrose-accessible pool. $\boldsymbol{A}$, The areas of EPSCS (open bar) and sucrose responses (filled middle bar) were measured at $0.5 \mathrm{~s}$ after high-frequency stimulation ( 100 pulses at $20 \mathrm{~Hz}$ ) and compared with the areas of original responses. ${ }^{*} p<0.001$ ( $n=9$ for EPSCs and 8 for sucrose). In separate cells, the sucrose response $0.5 \mathrm{~s}$ after a 40 pulse, $20 \mathrm{~Hz}$ train $(n=10)$ showed $\sim 25 \%$ depression, consistent with RRP discrepancies in Figure 3. $\boldsymbol{B}$, The difference in inhibition at various times after 100 stimuli at $20 \mathrm{~Hz}$ ( $n=3$ for each point). Recovery from inhibition was fit with a single exponential; $\tau=1.09 \mathrm{~s}$ (EPSCs; open circles) and $1.28 \mathrm{~s}$ (sucrose; filled circles). Fits were constrained to a maximum of $100 \%$. C, The areas of paired EPSCs (open circles) and sucrose responses (filled circles) were measured at various times after an initial pair of responses ( $n=5$ for each point). Recovery from inhibition was fit with a single exponential; $\tau=9.94 \mathrm{~s}$ (EPSCs) and $7.85 \mathrm{~s}$ (sucrose). Fits were constrained to a maximum of $100 \%$. Error bars represent SEM.
A
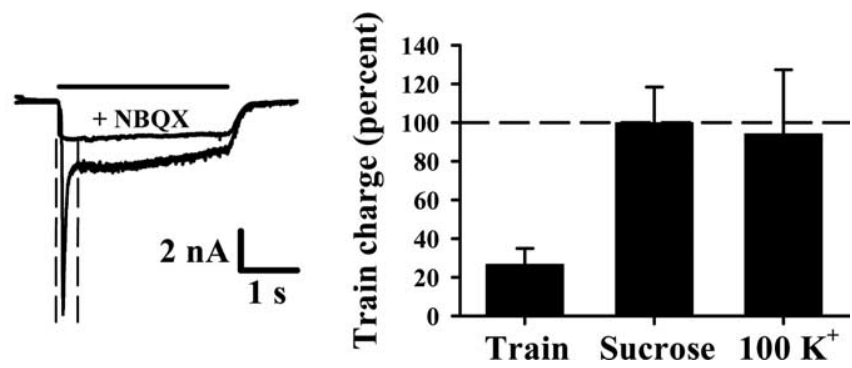

Figure 5. RRP size estimates using strong depolarizations match sucrose estimates. $\boldsymbol{A}$, Representative responses to hyperkalemic solution $\left(100 \mathrm{~mm} \mathrm{~K}^{+}\right)$application in the presence of 200 $\mu \mathrm{m}$ kynurenate, shown in the presence and absence of $1 \mu \mathrm{M}$ NBQX. Dashed lines indicate the portion of the response used to estimate RRP size, with the NBQX-insensitive component subtracted out in the analysis. $\boldsymbol{B}$, Summary of train, sucrose, or strong depolarization $\left(100 \mathrm{~K}^{+}\right)$ estimates of RRP size, expressed as percentages of the sucrose estimates of RRP size performed in the same neurons $(n=13)$. Error bars represent SEM.

vesicle recycling (Stevens and Wesseling, 1998) or perhaps that hypertonicity impedes RRP replenishment.

One interpretation of the experiments in Figure 4 is that action potentials release only a subset of vesicles in the RRP, because mechanisms other than full RRP depletion account for the apparent EPSC steady-state depression during trains. This would imply that some RRP vesicles, perhaps because of spatial or temporal heterogeneity in $p_{\mathrm{r}}$ values, are reluctant to release during action potential trains (Sun and $\mathrm{Wu}, 2001$ ). At the calyx of Held, strong direct depolarization of the presynaptic terminal releases vesicles that are not released in response to action potential trains (Schneggenburger et al., 2002). To test whether this is also true of hippocampal synapses, we used brief, acute applications of 100 mM K ${ }^{+}$(substituted for $\mathrm{Na}^{+}$) to evoke transmitter release by direct depolarization of terminals (Prakriya and Mennerick, 2000; Mozhayeva et al., 2002; Moulder et al., 2004). Hyperkalemic solution application resulted in an initial rapid peak response and a secondary steady-state component (Fig. 5A), which are thought to represent the release of the RRP and the reserve pool, respectively (Mozhayeva et al., 2002). The charge of the peak, NBQX-sensitive hyperkalemic response was therefore compared with the charge of the sucrose response and with the 40 stimuli, 20 $\mathrm{Hz}$ train estimates of RRP size in the same neurons. In the presence of kynurenate, RRP size estimates using strong depolarization matched sucrose estimates (Fig. 5B), consistent with the idea that sucrose-evoked vesicle release simply accesses all docked vesicles regardless of their individual $p_{\mathrm{r}}$ value and that strong prolonged depolarization is capable of accessing this same pool.

If reluctant vesicles do contribute to the total RRP in hippocampal neurons, a longer stimulus train should eventually be able to induce the release of these vesicles, even if they have a lower $p_{\mathrm{r}}$ value. To explore this possibility, we increased the number of stimuli delivered in $20 \mathrm{~Hz}$ trains from 40 to 100 . The decay in EPSC amplitude could be fit with a double exponential (Fig. $6 A$ ), suggesting multiple populations of vesicles with differing $p_{\mathrm{r}}$ values (Hessler et al., 1993; Rosenmund et al., 1993). Cumulative area plots were then made from the 100 stimuli trains, with linear extrapolations back to time 0 made from 1.5 to $2 \mathrm{~s}$ into the train (as in Figs. 1-3) and from 4.5 to $5 \mathrm{~s}$ (Fig. $6 \mathrm{~B}$ ). Train estimates of pool size derived from all 100 stimuli were, on average, 1.86 times larger than estimates derived from only 40 stimuli (Fig. 6C), consistent with the idea that the release of vesicles with a lower $p_{\mathrm{r}}$ 


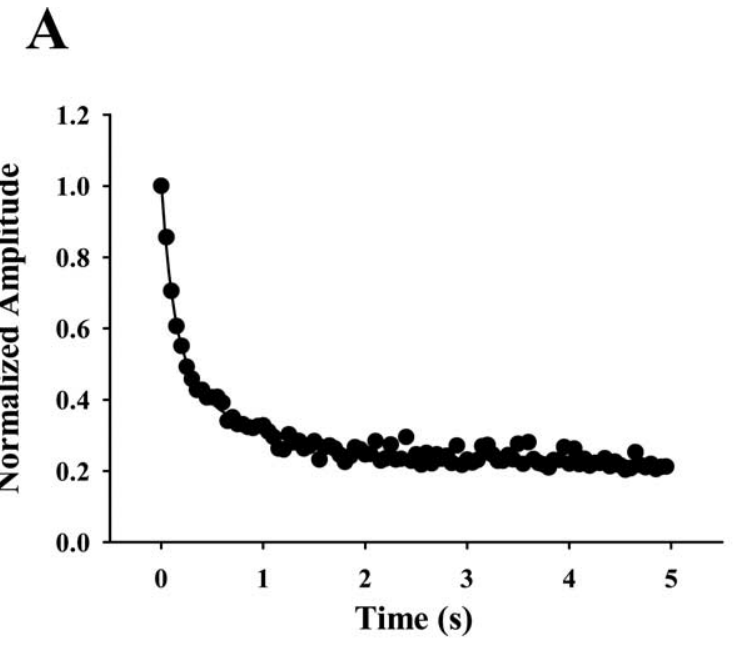

\section{B}
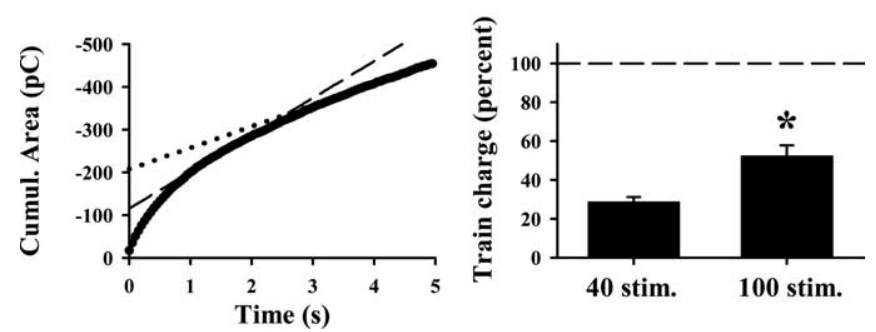

Figure 6. Increasing $20 \mathrm{~Hz}$ trains to 100 stimuli suggests multiple components of EPSC depression and a greater estimate of RRP size. $A$, Peak EPSC amplitude values, measured in the presence of $200 \mu \mathrm{m}$ kynurenate, during 100 stimuli, $20 \mathrm{~Hz}$ trains, normalized to the initial peak amplitude to illustrate the amplitude depression $(n=8)$. The normalized amplitudes were fit with a double-exponential decay; $\tau_{1}=122 \mathrm{~ms} ; \tau_{2}=773 \mathrm{~ms}$. $\boldsymbol{B}$, Representative cumulative EPSC area (Cumul. Area) values from a 100 stimuli, $20 \mathrm{~Hz}$ train. The dashed line represents a linear regression fit to time 0 from data points between 1.5 and $2 \mathrm{~s}$, and the dotted line represents a linear regression fit to time 0 from data points between 4.5 and $5 \mathrm{~s}$. C, Summary of the total RRP charge, as determined with $20 \mathrm{~Hz}$ trains of 40 or 100 stimuli (stim.), expressed as a percentage of the sucrose estimates of RRP size performed in the same neurons; ${ }^{*} p<0.001$ compared with estimates derived from 40 stimuli $(n=8)$. Error bars represent SEM.

value can be elicited with an increased number of stimuli. However, even estimates of pool size made using 100 stimuli were significantly smaller than sucrose estimates of RRP size made in the same neurons. Also, we cannot exclude the possibility that the slow components of release reflect the replenishment of the RRP rather than sluggish RRP depletion. Nevertheless, this experiment questions one of the basic assumptions of the action potential method: that a steady-state level of depletion is reached during short trains of $20 \mathrm{~Hz}$ stimulation.

If the slow phase of release during action potential trains represents vesicles with a low $p_{\mathrm{r}}$ value, consequently, by raising the overall $p_{\mathrm{r}}$ value with increased $\mathrm{Ca}^{2+}$ influx, we would expect a better match between the action potential and sucrose estimates of RRP. Forty stimuli at $20 \mathrm{~Hz}$ were delivered to neurons in the presence of 2 and $4 \mathrm{mM} \mathrm{Ca}^{2+}$, and the train estimates of RRP size were compared with sucrose responses made with the same extracellular calcium concentration $\left(\left[\mathrm{Ca}^{2+}\right]_{\mathrm{o}}\right)$. A 4 mM concentration of $\mathrm{Ca}^{2+}$ was chosen because we showed previously that this is near the peak of the $\left[\mathrm{Ca}^{2+}\right]_{\mathrm{o}}$-EPSC relationship (Moulder et al., 2003). As anticipated, the size of sucrose responses was unchanged by increasing $\left[\mathrm{Ca}^{2+}\right]_{\mathrm{o}}(99.8 \pm 5.8 \%$ of the response measured in $2 \mathrm{mM} \mathrm{Ca}^{2+}$ ). In contrast, train estimates of RRP

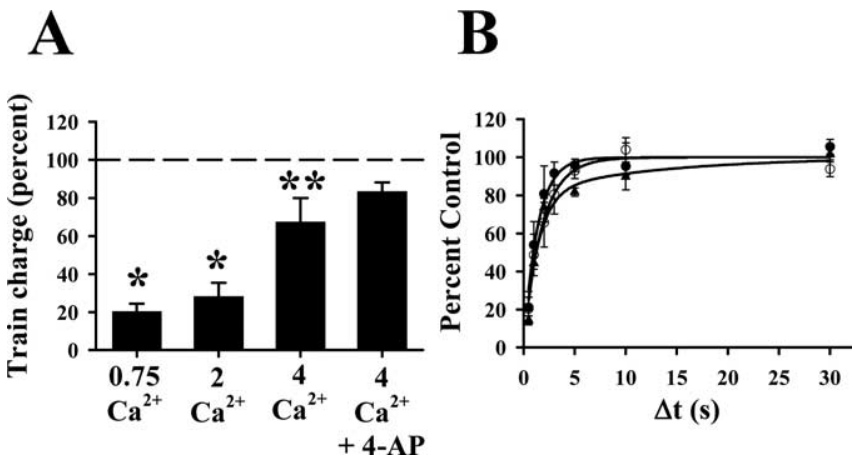

Figure 7. Raising $\left[\mathrm{Ca}^{2+}\right]_{0}$ and increasing action potential duration increase train estimates of RRP size. $A$, Summary of the total RRP charge, as determined with 40 stimuli, $20 \mathrm{~Hz}$ trains recorded in the presence of $0.75 \mathrm{~mm} \mathrm{Ca}^{2+}, 2 \mathrm{mM} \mathrm{Ca}^{2+}, 4 \mathrm{~mm} \mathrm{Ca}^{2+}$, or $4 \mathrm{~mm} \mathrm{Ca}^{2+}$ plus $100 \mu \mathrm{m}$ 4-AP, expressed as a percentage of the sucrose estimates of RRP size performed in the same neurons; ${ }^{*} p<0.002$ and $^{* *} p<0.042$ compared with sucrose estimates of RRP size $(n=5-14$ in each condition). $\boldsymbol{B}$, The areas of EPS(s were measured at various times after high-frequency stimulation in $0.75 \mathrm{~mm} \mathrm{Ca}^{2+}$ (filled triangles), $2 \mathrm{~mm} \mathrm{Ca}^{2+}$ (filled circles), and $4 \mathrm{~mm} \mathrm{Ca}^{2+}$ (open circles) and compared with the areas of original responses ( $n=4-5$ for each point). Recovery from inhibition was fit with a double exponential; weighted $\tau=3.49 \mathrm{~s}\left(0.75 \mathrm{~mm} \mathrm{Ca}{ }^{2+}\right), 1.37 \mathrm{~s}$ $\left(2 \mathrm{~mm} \mathrm{Ca}^{2+}\right)$, and $1.82 \mathrm{~s}\left(4 \mathrm{~mm} \mathrm{Ca}^{2+}\right)$. Fits were constrained to a maximum of $100 \%$. Error bars represent SEM.

were increased to $67 \%$ of sucrose estimates of RRP size (Fig. 7A), although this difference was still statistically significant. We next asked whether increasing action potential duration, and therefore driving more $\mathrm{Ca}^{2+}$ into terminals, could further increase train estimates of RRP size. We inhibited voltage-gated $\mathrm{K}^{+}$channels with $100 \mu \mathrm{M} 4$-aminopyridine (4-AP) in the presence of 4 $\mathrm{mM} \mathrm{Ca}^{2+}$. With 4-AP present, train estimates of RRP size were no longer statistically different from sucrose estimates made in the same cells under the same recording conditions (Fig. 7A). This additional increase in train estimates of RRP size with 4-AP was not attributable to a sustained presynaptic inward current caused by 4-AP application itself, because similar results were obtained when EPSC trains were NBQX subtracted (data not shown).

The increase in train estimates of RRP size with $4 \mathrm{mM} \mathrm{Ca}^{2+}$ likely resulted in part from a greater increase in asynchronous vesicle release than synchronous release, consistent with previous reports (Hagler and Goda, 2001). Measures of the EPSC cumulative area, which would account for asynchronous release, increased more after switching to $4 \mathrm{mM} \mathrm{Ca}^{2+}$ than measures of EPSC cumulative amplitude, which would only represent synchronous release; average cumulative area increased 2.39 times, whereas average cumulative amplitude only increased 1.69 times with the switch from 2 to $4 \mathrm{mM} \mathrm{Ca}^{2+}$. Asynchrony is also likely to increase in the presence of 4-AP because of the widening of the presynaptic action potential.

Because the $\mathrm{Ca}^{2+}$ influx that accompanies action potential trains may influence RRP replenishment (Stevens and Wesseling, 1998; Wang and Kaczmarek, 1998), we wanted to verify that the increases in RRP size estimates that we observed with $4 \mathrm{mM} \mathrm{Ca}^{2+}$ did not simply result from enhanced vesicle replenishment under these conditions. To do this, experiments similar to those in Figure $4 B$ were performed, in which 100 stimuli were delivered at 20 $\mathrm{Hz}$, and the recovery of EPSC area was examined in either $2 \mathrm{mM}$ $\mathrm{Ca}^{2+}$ or $4 \mathrm{mM} \mathrm{Ca}^{2+}$ plus $100 \mu \mathrm{M} 4$-AP. The decrease in the amount of inhibition after the action potential train was fit with a single exponential in each condition. Recovery of the EPSC area was slightly slower in the presence of $4 \mathrm{mM} \mathrm{Ca}^{2+}$ plus $100 \mu \mathrm{M}$ 4 -AP with a time constant of $1.75 \mathrm{~s}$ compared with $1.05 \mathrm{~s}$ in the presence of $2 \mathrm{mM} \mathrm{Ca}^{2+}$ (Fig. $7 B$ ). 
These values of recovery from train-induced depression were faster than we observed previously with recovery from singlepulse depression (Mennerick and Zorumski, 1995), suggesting that $\mathrm{Ca}^{2+}$ accumulation may speed recovery from depression. However, the difference between trains in normal $\mathrm{Ca}^{2+}$ and elevated $\mathrm{Ca}^{2+}$ plus 4 -AP was not statistically significant and was the opposite of what one would expect if enhanced vesicle replenishment contributed to train estimates of RRP size in $4 \mathrm{mM} \mathrm{Ca}^{2+}$. Similarly, estimates of replenishment obtained from the slope of cumulative release plots (Fig. $1 D$ ) suggested that replenishment was, if anything, slower during the train itself in the presence of 4 $\mathrm{mM} \mathrm{Ca}^{2+}$ plus 4 -AP (estimated at a rate of $19.1 \%$ of the pool per second during the steady-state phase of trains) compared with the recovery depicted in Figure $7 B$ (estimated initial rate of $\sim 57.2 \%$ of the pool per second in the first $50 \mathrm{~ms}$ of recovery). Finally, we also examined the pool size estimates and pulse-chase replenishment in the presence of low $\left[\mathrm{Ca}^{2+}\right]_{\mathrm{o}}$ (Fig. 7). Consistent with the high- $\mathrm{Ca}^{2+}$ data, we found that pool estimates were depressed (Fig. 7A) despite a slight slowing of replenishment rate relative to high- $\mathrm{Ca}^{2+}$ conditions (Fig. $7 B$ ). These results again are evidence of a dissociation between replenishment rate and pool size estimates. Together, the above results suggest that reluctant vesicle recruitment, rather than enhanced mobilization, accounts for the matched action potential and sucrose RRP estimates under these conditions.

\section{Discussion}

The size of the RRP is a critical factor determining the size of a PSC in response to a presynaptic action potential. Accordingly, modulation of RRP size represents a fundamental means by which synaptic strength and synaptic plasticity are altered. For example, changes in electrical activity, as well as exposure to neurotrophic factors, alter RRP size or, ultrastructurally, the number of docked vesicles (Collin et al., 2001; Murthy et al., 2001; Tyler and Pozzo-Miller, 2001; Moulder et al., 2004). Therefore, accurately measuring the size of the RRP is important for understanding vesicle availability under normal conditions and during the course of synaptic plasticity.

We found that two common methods for determining RRP size, application of hypertonic sucrose solution and highfrequency action potential trains, yielded very different RRP size estimates in glutamatergic hippocampal neurons. When these two methods were performed in the same neurons, sucrose estimates were consistently five to six times greater than estimates derived from action potential trains, even if the total pool charge was used as the common measurement in each (Fig. 1). AMPA receptor saturation appeared to play a small role in this discrepancy (Fig. 3). The majority of the effect was presynaptic. Raising $\left[\mathrm{Ca}^{2+}\right]_{0}$ and lengthening action potential duration increased train estimates of RRP size (Fig. 7A), consistent with the idea that reluctant synaptic vesicles contribute to the total RRP.

Depression of EPSCs during a train is typically assumed to result from the depletion of the available synaptic vesicles (Elmqvist and Quastel, 1965). This should be true under conditions in which depletion occurs more rapidly than recovery (such as with high-frequency stimulation) (Schneggenburger et al., 1999). However, an immediate conclusion from our data is that protocols of short action potential trains at moderate frequencies $(20-40 \mathrm{~Hz})$, commonly used in the literature as a depleting stimulus (Murthy and Stevens, 1998; Pyle et al., 2000; Schikorski and Stevens, 2001), are unlikely to deplete the total available RRP in the presence of physiological $\left[\mathrm{Ca}^{2+}\right]_{\mathrm{o}}(2 \mathrm{mM})$. Our results apparently contradict previous results in the same preparation (Rosen- mund and Stevens, 1996), in which short action potential trains at $20 \mathrm{~Hz}$ were found to deplete the sucrose-accessible RRP completely. Part of the difference in results may come from the failure to strip away the steady-state EPSC responses during action potential trains in the older work, which would result in inflated action potential RRP estimates (Schneggenburger et al., 2002). It is also possible that slightly higher $\left[\mathrm{Ca}^{2+}\right]_{\mathrm{o}}$ may have resulted in a higher overall $p_{\mathrm{r}}$ value in the older work. Even so, in our study, short $20 \mathrm{~Hz}$ trains yielded an apparent steady state (Fig. 1) that did not truly reflect full depletion, because RRP measures under these conditions did not match estimates from strong direct depolarization or from trains with enhanced $\mathrm{Ca}^{2+}$ influx. These results clearly raise a caution that steady-state EPSC depression at hippocampal synapses does not necessarily equate with full RRP depletion. Although we were surprised at the magnitude of the discrepancy between the two depletion methods, our estimates of $\mathrm{RRP}$ charge (158 pC for action potential trains and $1034 \mathrm{pC}$ for sucrose) (Fig. 1) match very closely with previously published values (Han et al., 2004; Otsu et al., 2004), suggesting that RRP mismatch is a widespread issue.

Cross-depletion experiments, in which high-frequency stimulation or sucrose application was used to deplete the RRP (Fig. 4 ), suggested that action potential trains accessed a smaller fraction of the same total pool that was depleted by sucrose. The mechanism by which sucrose induces release of the RRP remains poorly understood, with the exception that it is known to be $\mathrm{Ca}^{2+}$ independent (Fatt and Katz, 1952; Rosenmund and Stevens, 1996) and perhaps related to actions on integrins (Grinnell et al., 2003). For this reason, it might be argued that sucrose estimates of the RRP are likely inaccurate, reflecting vesicles that are not truly part of the RRP. In an alternative interpretation of our results, hyperkalemia and enhanced $\mathrm{Ca}^{2+}$ influx may have recruited vesicles from the sucrose-accessible pool and enlarged the "true" RRP. Although we cannot fully exclude this alternative, we prefer the explanation that strong direct depolarization and enhanced $\mathrm{Ca}^{2+}$ influx during trains simply increase the $p_{r}$ value, consistent with a wealth of data at many synapses (Katz and Miledi, 1968, 1970; Wang and Zucker, 1998; Stevens and Wesseling, 1999).

Another alternative explanation for our major findings is that sucrose and action potentials access fundamentally different pools of vesicles, but strong depolarization and enhanced $\mathrm{Ca}^{2+}$ influx speed replenishment of the action potential-accessible pool, so that the apparent size of the action potential pool eventually matches the sucrose pool. We found that recovery of EPSCs from train stimulation was significantly faster than recovery from single-pulse stimulation (Mennerick and Zorumski, 1995), consistent with $\mathrm{Ca}^{2+}$-dependent mechanisms of replenishment (Wang and Kaczmarek, 1998; Sara et al., 2002). However, we found that the replenishment rate was similar in trains evoked in physiological $\left[\mathrm{Ca}^{2+}\right]_{\mathrm{o}}$ compared with trains with chemically potentiated $\mathrm{Ca}^{2+}$ influx (Fig. 7). Therefore, the replenishment rate apparently reaches saturation during trains in $2 \mathrm{mM} \mathrm{Ca}^{2+}$ and cannot explain the increase in RRP observed in the two conditions (Fig. 7).

It is unclear in our experiments whether the $p_{\mathrm{r}}$ value is initially heterogeneous, perhaps resulting from spatial heterogeneity in $\left[\mathrm{Ca}^{2+}\right]_{\mathrm{i}}$ rises (Schneggenburger et al., 2002), or whether the $p_{\mathrm{r}}$ value actively changes with stimulation. Spatial heterogeneity has been suggested previously using a technique of low-frequency stimulation in the presence of an activation-dependent irreversible postsynaptic channel blocker (Hessler et al., 1993; Rosenmund et al., 1993). However, simple calculations in which all 
vesicles are assigned the lowest $p_{\mathrm{r}}$ value reported from these studies $\left(p_{\mathrm{r}}=0.06\right)$ still predicts an $\sim 92 \%$ depletion of the RRP over a 40 pulse train (data not shown). Even fuller depletion is predicted when heterogeneity is introduced from contributions of vesicles with higher $p_{\mathrm{r}}$ values (Hessler et al., 1993; Rosenmund et al., 1993). Therefore, it is possible that the $p_{\mathrm{r}}$ value does actively change with high-frequency stimulation, as suggested by previous studies (Betz, 1970; Dobrunz and Stevens, 1997; Silver et al., 1998; Wu and Borst, 1999; Chen et al., 2004). One possible mechanism that could account for such a change is the inactivation of presynaptic channels (Forsythe et al., 1998).

We also observed that the train estimates of RRP size closely matched sucrose estimates in GABAergic neurons, even in the presence of $2 \mathrm{mM} \mathrm{Ca}^{2+}$. This is consistent with the idea that GABAergic synapses contain vesicles with individual release probabilities that are higher and/or more homogeneous than those observed in glutamatergic neurons. Our observation that glutamate release may be subject to greater restraint than GABA release suggests that this mechanism may provide one means by which neurons can limit the possibility of detrimental effects from overexcitation or excitotoxicity. The heterogeneity among glutamate vesicles from the same presynaptic cell may also contribute to a greater potential for plasticity at these synapses, with modulators able to make vesicles more or less reluctant.

In sum, our results suggest that reluctant vesicles contribute to a heterogeneous RRP vesicle population in glutamatergic, but not GABAergic, hippocampal neurons. This heterogeneity may allow excitatory neurons to expand their range of synaptic output, depending on the strength of the incoming depolarization and the extracellular ion composition. This form of plasticity is a particularly attractive mechanism by which synaptic transmission is modulated, because an altered response simply makes use of vesicles that are already in place, without the need to recruit additional postsynaptic components.

\section{References}

Aravamudan B, Fergestad T, Davis WS, Rodesch CK, Broadie K (1999) Drosophila UNC-13 is essential for synaptic transmission. Nat Neurosci 2:965-971.

Betz WJ (1970) Depression of transmitter release at the neuromuscular junction of the frog. J Physiol (Lond) 206:629-644.

Brenowitz S, Trussell LO (2001) Minimizing synaptic depression by control of release probability. J Neurosci 21:1857-1867.

Brody DL, Yue DT (2000) Release-independent short-term synaptic depression in cultured hippocampal neurons. J Neurosci 20:2480-2494.

Chen G, Harata NC, Tsien RW (2004) Paired-pulse depression of unitary quantal amplitude at single hippocampal synapses. Proc Natl Acad Sci USA 101:1063-1068.

Collin C, Vicario-Abejon C, Rubio ME, Wenthold RJ, McKay RD, Segal M (2001) Neurotrophins act at presynaptic terminals to activate synapses among cultured hippocampal neurons. Eur J Neurosci 13:1273-1282.

Diamond JS, Jahr CE (1995) Asynchronous release of synaptic vesicles determines the time course of the AMPA receptor-mediated EPSC. Neuron 15:1097-1107.

Diamond JS, Jahr CE (1997) Transporters buffer synaptically released glutamate on a submillisecond time scale. J Neurosci 17:4672-4687.

Dobrunz LE, Stevens CF (1997) Heterogeneity of release probability, facilitation, and depletion at central synapses. Neuron 18:995-1008.

Elmqvist D, Quastel DM (1965) A quantitative study of end-plate potentials in isolated human muscle. J Physiol (Lond) 178:505-529.

Fatt P, Katz B (1952) Spontaneous subthreshold activity at motor nerve endings. J Physiol (Lond) 117:109-128.

Forsythe ID, Tsujimoto T, Barnes-Davies M, Cuttle MF, Takahashi T (1998) Inactivation of presynaptic calcium current contributes to synaptic depression at a fast central synapse. Neuron 20:797-807.

Foster KA, Kreitzer AC, Regehr WG (2002) Interaction of postsynaptic re- ceptor saturation with presynaptic mechanisms produces a reliable synapse. Neuron 36:1115-1126.

Grinnell AD, Chen BM, Kashani A, Lin J, Suzuki K, Kidokoro Y (2003) The role of integrins in the modulation of neurotransmitter release from motor nerve terminals by stretch and hypertonicity. J Neurocytol 32:489-503.

Hagler Jr DJ, Goda Y (2001) Properties of synchronous and asynchronous release during pulse train depression in cultured hippocampal neurons. J Neurophysiol 85:2324-2334.

Han W, Rhee JS, Maximov A, Lao Y, Mashimo T, Rosenmund C, Sudhof TC (2004) N-glycosylation is essential for vesicular targeting of synaptotagmin 1. Neuron 41:85-99.

He Y, Zorumski CF, Mennerick S (2001) Contribution of presynaptic $\mathrm{Na}^{+}$ channel inactivation to paired-pulse synaptic depression in cultured hippocampal neurons. J Neurophysiol 87:925-936.

Hessler NA, Shirke AM, Malinow R (1993) The probability of transmitter release at a mammalian central synapse. Nature 366:569-572.

Johnson EW, Wernig A (1971) The binomial nature of transmitter release at the crayfish neuromuscular junction. J Physiol (Lond) 218:757-767.

Katz B (1969) The release of neural transmitter substances. Liverpool, UK: Liverpool UP.

Katz B, Miledi R (1968) The role of calcium in neuromuscular facilitation. J Physiol (Lond) 195:481-492.

Katz B, Miledi R (1970) Further study of the role of calcium in synaptic transmission. J Physiol (Lond) 207:789-801.

Kirischuk S, Grantyn R (2000) A readily releasable pool of single inhibitory boutons in culture. NeuroReport 11:3709-3713.

Lu T, Trussell LO (2000) Inhibitory transmission mediated by asynchronous transmitter release. Neuron 26:683-694.

Mennerick S, Zorumski CF (1995) Paired-pulse modulation of fast excitatory synaptic currents in microcultures of rat hippocampal neurons. J Physiol (Lond) 488:85-101.

Mennerick S, Que J, Benz A, Zorumski CF (1995) Passive and synaptic properties of hippocampal neurons grown in microcultures and in mass cultures. J Neurophysiol 73:320-332.

Moulder KL, Cormier RJ, Shute AA, Zorumski CF, Mennerick S (2003) Homeostatic effects of depolarization on $\mathrm{Ca}^{2+}$ influx, synaptic signaling, and survival. J Neurosci 23:1825-1831.

Moulder KL, Meeks JP, Shute AA, Hamilton CK, de Erausquin G, Mennerick S (2004) Plastic elimination of functional glutamate release sites by depolarization. Neuron 42:423-435.

Mozhayeva MG, Sara Y, Liu X, Kavalali ET (2002) Development of vesicle pools during maturation of hippocampal synapses. J Neurosci 22:654-665.

Murthy VN, Stevens CF (1998) Synaptic vesicles retain their identity through the endocytic cycle. Nature 392:497-501.

Murthy VN, Stevens CF (1999) Reversal of synaptic vesicle docking at central synapses. Nat Neurosci 2:503-507.

Murthy VN, Schikorski T, Stevens CF, Zhu Y (2001) Inactivity produces increases in neurotransmitter release and synapse size. Neuron 32:673-682.

Neher E, Sakaba T (2001) Combining deconvolution and noise analysis for the estimation of transmitter release rates at the calyx of held. J Neurosci 21:444-461.

Otsu Y, Shahrezaei V, Li B, Raymond LA, Delaney KR, Murphy TH (2004) Competition between phasic and asynchronous release for recovered synaptic vesicles at developing hippocampal autaptic synapses. J Neurosci 24:420-433.

Prakriya M, Mennerick S (2000) Selective depression of low-release probability excitatory synapses by sodium channel blockers. Neuron 26:671-682.

Pyle JL, Kavalali ET, Piedras-Renteria ES, Tsien RW (2000) Rapid reuse of readily releasable pool vesicles at hippocampal synapses. Neuron 28:221-231.

Quastel DM (1997) The binomial model in fluctuation analysis of quantal neurotransmitter release. Biophys J 72:728-753.

Reim K, Mansour M, Varoqueaux F, McMahon HT, Sudhof TC, Brose N, Rosenmund C (2001) Complexins regulate a late step in $\mathrm{Ca}^{2+}$. dependent neurotransmitter release. Cell 104:71-81.

Rosenmund C, Stevens CF (1996) Definition of the readily releasable pool of vesicles at hippocampal synapses. Neuron 16:1197-1207. 
Rosenmund C, Clements JD, Westbrook GL (1993) Nonuniform probability of glutamate release at a hippocampal synapse. Science 262:754-757.

Sara Y, Mozhayeva MG, Liu X, Kavalali ET (2002) Fast vesicle recycling supports neurotransmission during sustained stimulation at hippocampal synapses. J Neurosci 22:1608-1617.

Schikorski T, Stevens CF (2001) Morphological correlates of functionally defined synaptic vesicle populations. Nat Neurosci 4:391-395.

Schneggenburger R, Meyer AC, Neher E (1999) Released fraction and total size of a pool of immediately available transmitter quanta at a calyx synapse. Neuron 23:399-409.

Schneggenburger R, Sakaba T, Neher E (2002) Vesicle pools and short-term synaptic depression: lessons from a large synapse. Trends Neurosci 25:206-212.

Silver RA, Momiyama A, Cull-Candy SG (1998) Locus of frequencydependent depression identified with multiple-probability fluctuation analysis at rat climbing fibre-Purkinje cell synapses. J Physiol (Lond) 510:881-902.

Stevens CF, Wesseling JF (1998) Activity-dependent modulation of the rate at which synaptic vesicles become available to undergo exocytosis. Neuron 21:415-424.

Stevens CF, Wesseling JF (1999) Augmentation is a potentiation of the exocytotic process. Neuron 22:139-146.
Sun JY, Wu LG (2001) Fast kinetics of exocytosis revealed by simultaneous measurements of presynaptic capacitance and postsynaptic currents at a central synapse. Neuron 30:171-182.

Tong G, Jahr CE (1994) Block of glutamate transporters potentiates postsynaptic excitation. Neuron 13:1195-1203.

Tyler WJ, Pozzo-Miller LD (2001) BDNF enhances quantal neurotransmitter release and increases the number of docked vesicles at the active zones of hippocampal excitatory synapses. J Neurosci 21:4249-4258.

Wadiche JI, Jahr CE (2001) Multivesicular release at climbing fiberPurkinje cell synapses. Neuron 32:301-313.

Wang C, Zucker RS (1998) Regulation of synaptic vesicle recycling by calcium and serotonin. Neuron 21:155-167.

Wang LY, Kaczmarek LK (1998) High-frequency firing helps replenish the readily releasable pool of synaptic vesicles. Nature 394:384-388.

Wu LG, Borst JG (1999) The reduced release probability of releasable vesicles during recovery from short-term synaptic depression. Neuron 23:821-832.

Xu-Friedman MA, Regehr WG (2003) Ultrastructural contributions to desensitization at cerebellar mossy fiber to granule cell synapses. J Neurosci 23:2182-2192.

Zucker RS (1973) Changes in the statistics of transmitter release during facilitation. J Physiol (Lond) 229:787-810. 